Calculus from the past: Multiple Delay Systems arising in Cancer Cell Modelling

by

\author{
G. C. Wake \\ H. M. Byrne
}





\title{
CALCULUS FROM THE PAST: MULTIPLE DELAY SYSTEMS ARISING IN CANCER CELL MODELLING
}

\author{
G. C. WAKE ${ }^{1} \quad$ H. M BYRNE ${ }^{2}$
}

November 9, 2012

\begin{abstract}
Non-local calculus is often overlooked in the mathematics curriculum. In this paper we present an interesting new class of non-local problems that arise from modelling the growth and division of cells, especially cancer cells, as they progress through the cell cycle. The cellular biomass is assumed to be unstructured in size or position, and its evolution governed by a time-dependent system of ordinary differential equations with multiple time delays. The system is linear and taken to be autonomous. As a result, it is possible to reduce its solution to that of a nonlinear matrix eigenvalue problem. This method is illustrated by considering case studies, including the model of the cell cycle developed in Simms K, Bean N, \& Koeber A. [10]. The paper concludes by explaining how asymptotic expressions for the distribution of cells across the compartments can be determined and used to assess the impact of different chemotherapeutic agents.
\end{abstract}

\section{Contents}

1 Introduction $\quad 2$

2 A simple example $\quad 2$

3 Multiple delays : a case-study 4

3.1 Model Development . . . . . . . . . . . . . . . . . 5

3.2 Model simplification and analysis . . . . . . . . . . . 7

3.3 Full System . . . . . . . . . . . . . . . . 7 
$\begin{array}{lll}4 \text { Conclusion } & 11\end{array}$

$\begin{array}{lr}\text { References } & 12\end{array}$

\section{Introduction}

Non-local calculus is a general term used to describe situations (arising in models) where cause and effect are separated explicitly in time (a delay), space, age, or even size, depending on how the cohort or state variables are structured. The simplest case considered here is a time-delay where the current evolutionary dynamics depend, in an explicit way, on the state of the system at an earlier time. We consider this situation here, albeit in a system which has many compartments.

Models can have point delays or these can be distributed over earlier intervals. The former have a long history, see for example Bellman and Cooke [4]. In the second case, which in $[6,8]$ are called distributed-delaydifferential equations (DDDEs), one can often eliminate the non-local effect by a simple transformation. Point delays generally resist this and thereby preserve their capacity to model simply quite complex behaviour. In this paper we focus on point time-delays for linear equations. We provide a generic procedure for obtaining an exact analytic solution (these multiple delay linear systems then need some simple numerical analysis to determine the detailed solution). In particular, when applied to the multistage cellcycle model in $[1,2,3]$ our method provides asymptotic expressions for the proportions of cells in the different cell cycle compartments. These results constitute a useful underpinning procedure for evaluating experimentally the effectiveness of potential new drugs for chemical treatment of cancer cells, when they are administrated 'in vitro' [7].

\section{A simple example}

The classic textbook problem, see [4]

$$
y^{\prime}(t)=y(t-T), t, T>0
$$

illustrates nicely the richness that delay problems exhibit. This is particularly useful when one is seeking a suitably simple formulation for modelling complex phenomena. In particular, the spanning set of equation (1) is the countably infinite set 


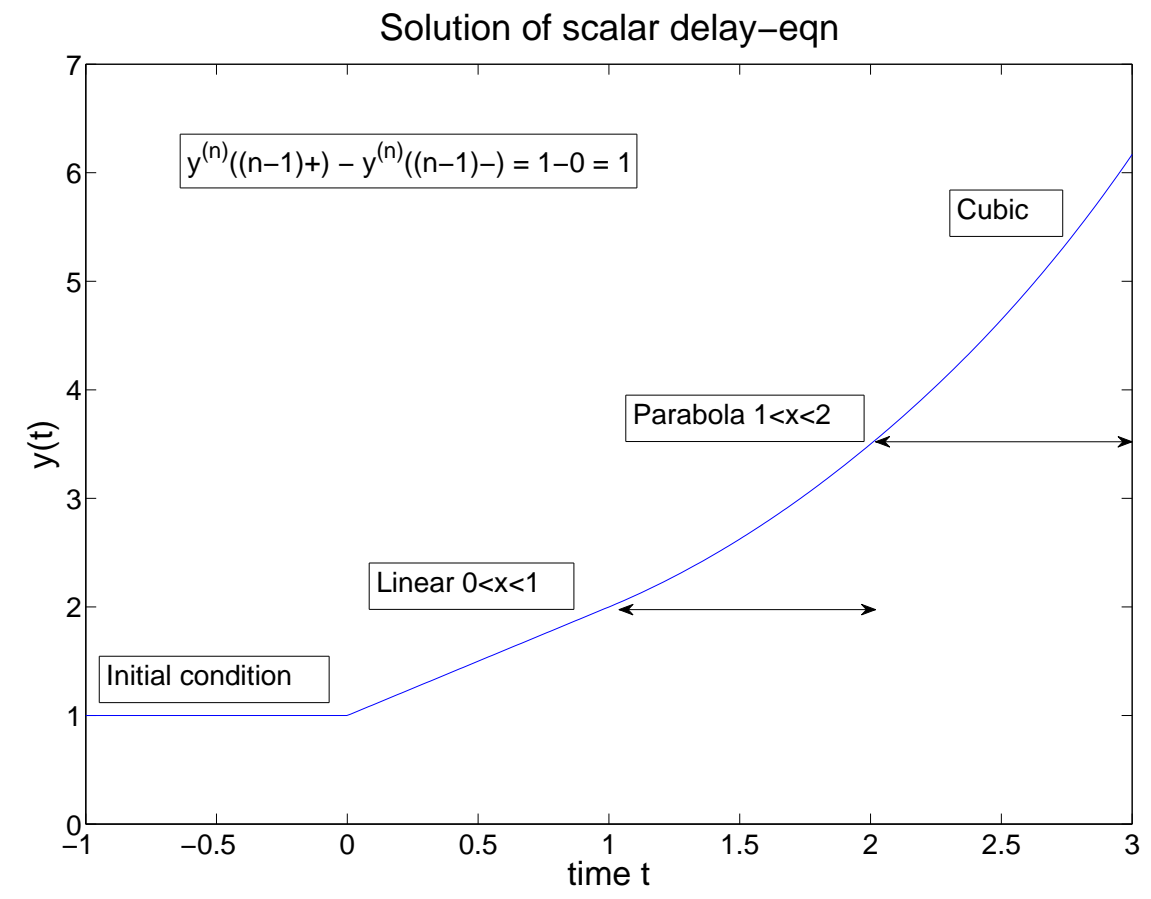

Figure 1: Solution of equation (1) with unit step time-delay and constant initial condition for $-1 \leq t \leq 0$ obtained by iteration, over successive time-intervals.

$$
\Omega=\left\{e^{\lambda t}: \lambda \in \Lambda\right\} \text { where } \Lambda=\left\{\lambda: e^{-\lambda T}=\lambda\right\} .
$$

The equation in $\Lambda$ for $\lambda$ has only one real (positive) root $\lambda_{0}(=0.5671$ when $T=1$ ) and an infinite number of complex roots in complex conjugate pairs with negative real part. This enables the problem to be well-posed with an arbitrary initial condition

$$
y(t)=y_{0}(t),-T \leq t \leq 0 .
$$

(In contrast, when $T=0$, the spanning set is of one-dimension).

Clearly then, we have the asymptotic behaviour, for a

$$
y(t) \sim K_{0} e^{\lambda_{0} t} \text { for large } t,
$$

where the constant $K_{0}$ depends in an explicit way on $y_{0}(t)$. This result, which is easily obtained by Laplace transforms, is far from obvious from the piecewise integration of equation (1), which when $T=1$ and $y_{0}(t) \equiv 1$, gives $y(t)$ as a polynomial of degree $n$ in $n T<t<(n+T)$, with discontinuities in 
higher derivatives as $n$ increases at the end points. This is shown in Figure 1. The pattern shown there gives no suggestion that the long-term behaviour is as in equation (3). There is nothing here that suggests that $x(t) \sim e^{-0.57 t}$ for $t$ large.

The general solution of equation (1) is obtained by finding the set $\Lambda$ in equation (3). When $T=1$ this gives the transcendental equation $\lambda=e^{-\lambda}$. If we let $\lambda_{1}=\operatorname{Re}(\lambda), \lambda_{2}=\operatorname{Im}(\lambda)$ these satisfy the simultaneous equations

$$
\text { (i) } \lambda_{1}=e^{-\lambda_{1}} \cos \left(\lambda_{2}\right) \text {; and (ii) } \lambda_{2}=-e^{-\lambda_{1}} \sin \left(\lambda_{2}\right) \text {. }
$$

These loci are drawn in Figure 2, with (i) in red and (ii) in blue. The roots are thus the intersections of the red and blue lines.

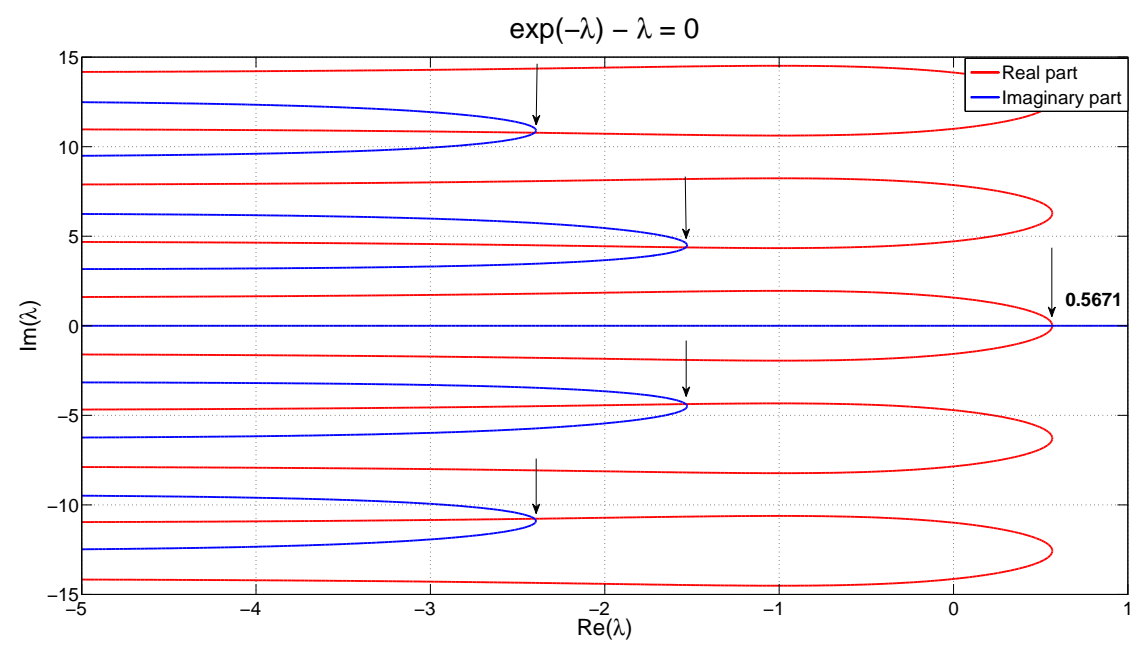

Figure 2: The roots of the transcendental equations in equation (4). The only roots shown are $\lambda=0.5671,-1.543 \pm 4.375 i,-2.402 \pm 10.776 i$.

\section{Multiple delays : a case-study}

There can be multiple delays in systems. In Section 3.1 we give an example from Cell-cycle population dynamics, from Simms et al [10]. This model can be generalised. We now give the general method for such a system. This is abstracted and written as

$$
\frac{d \boldsymbol{x}}{d t}=\boldsymbol{A} \boldsymbol{x}(t-[\boldsymbol{T}])
$$


where $\boldsymbol{x}(t)$ is a column vector of functions $\left\{x_{1}(t), \ldots x_{i}(t), \ldots \ldots x_{n}(t)\right\}, \boldsymbol{A}$ is a $n \times \mathrm{x} n$ (constant) matrix, $\boldsymbol{T}=\left(T_{i j}\right)$ and $[\boldsymbol{T}]=T_{i j}, j=1, \ldots n$ when $\mathbf{x} \rightarrow x_{i}$. This explains $[\boldsymbol{T}]$, that is, in component form, we have

$$
d x_{i} / d t=\sum_{j} a_{i j} x_{j}\left(t-T_{i j}\right)
$$

To solve equation (6) we let $\boldsymbol{x}(t)=\exp (\lambda t) \boldsymbol{c}$. This is analogous to that developed in Hale et al [5] but significantly different from their approach. Although they get the same sort of result. Then we have $\lambda \boldsymbol{c}=\boldsymbol{B}(\lambda) \boldsymbol{c}$, where $\boldsymbol{B}(\lambda)=a_{i j} e^{-\lambda T_{i j}}$. So

$$
\lambda \in\{\lambda: \operatorname{det}(\boldsymbol{B}(\lambda)-\lambda I)=0\}=\boldsymbol{\Lambda} .
$$

This is a "nonlinear in $\lambda$ " eigenvalue problem with a countable infinity of "eigenvalues", and $\boldsymbol{c}(\lambda)$ is the corresponding "eigenvector" of $\boldsymbol{B}(\lambda)$.

So the general solution of $d \boldsymbol{x} / d t=\boldsymbol{A x}(t-[\boldsymbol{T}])$, is therefore

$$
\boldsymbol{x}(t)=\sum_{\lambda \in \boldsymbol{\Lambda}} e^{\lambda t} \boldsymbol{c}(\lambda)
$$

We note the following properties which are inferred from examples only:

- If $T_{i j} \geq 0$ (as in the tumour model below), for all $i, j$; then there are a finite number of real eigenvalues, which are positive, and all others (an infinite number) are complex occurring in conjugate pairs, with smaller real part.

- Then, we get the asymptotic behaviour $\boldsymbol{x}(t) \sim e^{\lambda_{0} t} \boldsymbol{c}\left(\lambda_{0}\right)$ as $t \rightarrow \infty$, where $\lambda_{0}$ is the "eigenvalue" with largest real part.

- This gives as $x_{1}(t): x_{2}(t): \ldots: x_{n}(t) \sim c_{1}: c_{2}: \ldots: c_{n}$, as $t \rightarrow \infty$, that is, we have constant proportions asymptotically. This is important in applications, especially in relation to chemical therapy for cancer treatment.

\subsection{Model Development}

In cell cycling there are the usually three distinct populations of cells: the G1 (Gap, written as G), S (DNA replication) and G2/M (Gap/Mitosis, written as M)) phases. These are, in the model of Simms et al $[9,10]$, broken further into sub-compartments of storage and non-storage phases. The latter are the ones in which the cells are responsive to their environment. These are shown 
in the Figure below, from Simms et al [10].

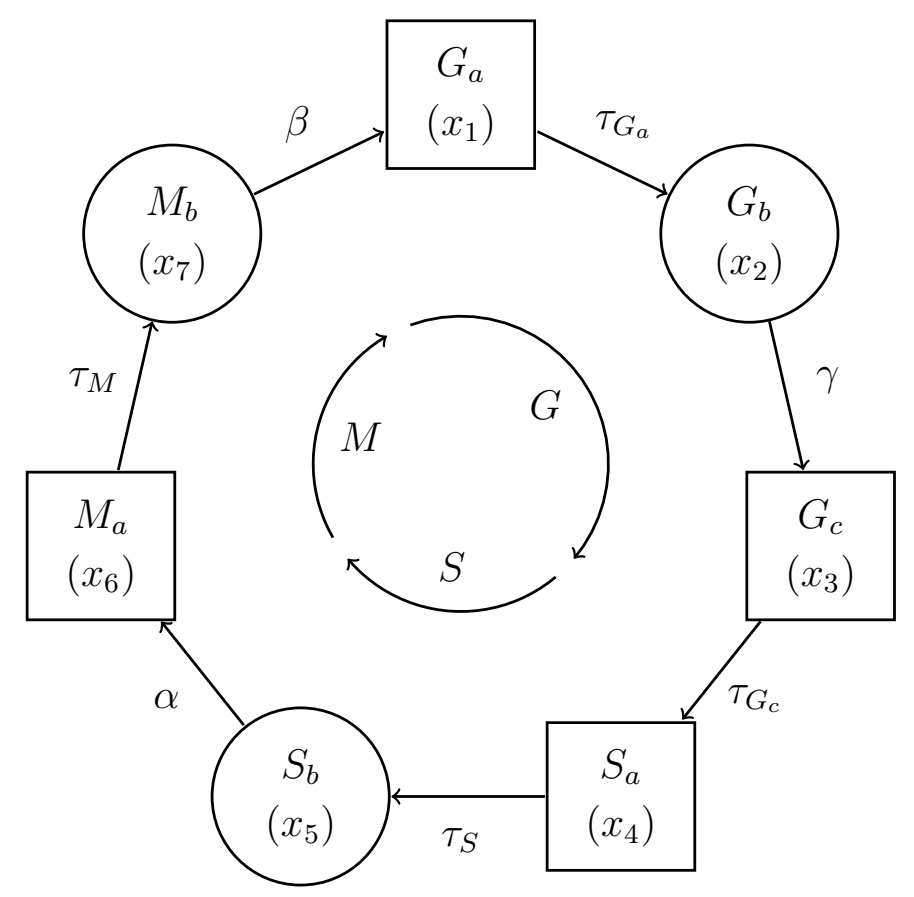

Figure 3: Cell cycle progression through the seven model phases. Each model phase is associated with an actual cell cycle phase (inner cyclic arrows). Model phases in square boxes represent storage phases and model phases in circles represent nonstorage phases. The parameters on arrows from storage phases represent the fixed time that a cell spends in that model phase, while the parameters on arrows from a non-storage phase represents the rate of transition from that phase.

This leads to the following system of equations:

Letting $N_{G_{a}}(t)=x_{1}(t), N_{G_{b}}(t)=x_{2}(t), \ldots, N_{M_{b}}(t)=x_{7}(t)$ represent the number of cells in the $G_{a}, G_{b}, \ldots, M_{b}$ phases at time $t$ respectively, and recalling that $\frac{d X(t)}{d t}$ represents the rate of change of $X(t)$ with respect to time, we write our model equations as follows:

$$
\begin{aligned}
\frac{d x_{1}}{d t} & =2 \beta x_{7}(t)-2 \beta x_{7}\left(t-\tau_{1}\right), \\
\frac{d x_{2}}{d t} & =2 \beta x_{7}\left(t-\tau_{1}\right)-\gamma x_{2}(t), \\
\frac{d x_{3}}{d t} & =\gamma x_{2}(t)-\gamma x_{2}\left(t-\tau_{3}\right),
\end{aligned}
$$




$$
\begin{aligned}
\frac{d x_{4}}{d t} & =\gamma x_{2}(t-\tau)-\gamma x_{2}\left(t-\tau_{3}-\tau_{s}\right) \\
\frac{d x_{5}}{d t} & =\gamma x_{2}\left(t-\tau_{3}-\tau_{s}\right)-\alpha x_{5}(t) \\
\frac{d x_{6}}{d t} & =\alpha x_{5}(t)-\alpha x_{5}\left(t-\tau_{M}\right) \\
\frac{d x_{7}}{d t} & =\alpha x_{5}\left(t-\tau_{M}\right)-\beta x_{7}(t)
\end{aligned}
$$

These equations fit the generic pattern embodied in equation (5). In the next two sections we will apply the method in the first part of Section 3 to solve this problem.

\subsection{Model simplification and analysis}

Close scrutiny of the seven equations in the above section show that all four of the storage phases $x_{1}, x_{3}, x_{4}$, and $x_{6}$ do not appear on the RHS and so the system is effectively driven by a smaller three-by-three system of equations (9b, 9e and 9f). Following the process outlined at the beginning of Section 3 the "eigenvalues" satisfy the transcendental equation

$$
\lambda^{3}+(\alpha+\beta+\gamma) \lambda^{2}+(\alpha \beta+\beta \gamma+\gamma \alpha) \lambda+\alpha \beta \gamma\left(1-2 e^{-\lambda\left(\tau_{1}+\tau_{2}+\tau_{3}+\tau_{4}\right)}\right)=0
$$

This could easily be solved and the corresponding eigenvectors obtained. However, we will not do this here as actually we need to find the fraction of cells in each of all seven compartments (including the storage compartments) which is able to be done for the full system in one step. We observe that $\lambda=0$ is not a solution of equations (9a-9g) showing there are no constant solutions of this core solution. Further there is just one real solution which is positive, and it will drive the dynamics of the solution in the long-term if the system is not perturbed. This will be shown in the next section where all of the equations will be included.

\subsection{Full System}

Here we use the same method to solve the whole system in the seven equations in $(9 \mathrm{a}-9 \mathrm{~g})$.

It is important to note, however, that the transcendental equation in equation (9a-9g) will be the same as that in equation (10) apart from the 
fact that $\lambda=0$ will be an "eigenvalue" of geometric multiplicity four showing that there are constant solutions (not growing in time) as well as possibly some with slower (algebraic) growth in time.

Secondly, by writing $X=\sum_{i} x_{i}$, for the total number of cells, we see on adding the seven equations in equations (9a-9g), we have

$$
d X / d t=\beta x_{7}
$$

So the total number of cells mirrors that of its constituent components with the same asymptotic behaviour.

Before proceeding to solve the full system by the same procedure, we observe that some of the components have more than one delay. There is one component $x_{2}$ which have all of: no delay and elsewhere has two different delays. This means we need to adapt the method above to a system of the form

$$
d \boldsymbol{x} / d t=\boldsymbol{A}^{(1)} \boldsymbol{x}\left(t-\left[T^{(1)}\right]+\boldsymbol{A}^{(2)} \boldsymbol{x}\left(t-\left[T^{(2)}\right]+\boldsymbol{A}^{(3)} \boldsymbol{x}\left(t-\left[T^{(3)}\right],\right.\right.\right.
$$

that is, there are three different "matrices of delay times". With values from the experimental situation [10] given in the following table:

\begin{tabular}{|c|c|}
\hline Symbol & Value and Units \\
\hline$\alpha$ & $0.6700 \mathrm{hr}^{-1}$ \\
$\beta$ & $2.3100 \mathrm{hr}^{-1}$ \\
$\gamma$ & $0.3284 \mathrm{hr}^{-1}$ \\
$\tau_{1}$ & $5.75 \mathrm{hrs}$ \\
$\tau_{2}$ & $1.00 \mathrm{hrs}$ \\
$\tau_{3}$ & $9.00 \mathrm{hrs}$ \\
$\tau_{4}$ & $3.40 \mathrm{hrs}$ \\
\hline
\end{tabular}

Table 1: Parameters from the Cancer Cell lines in Simms et al [10].

This gives the six very sparse seven-by-seven matrices below. 


\begin{tabular}{|c|c|c|c|c|c|c|c|}
\hline & 0 & 0 & 0 & 0 & 0 & 0 & 4.6200 \\
\hline & 0 & -0.3284 & 0 & 0 & 0 & 0 & 0 \\
\hline & 0 & 0.3284 & 0 & 0 & 0 & 0 & 0 \\
\hline$A_{1}=$ & 0 & 0 & 0 & 0 & 0 & 0 & 0 \\
\hline & 0 & 0 & 0 & 0 & -0.6700 & 0 & 0 \\
\hline & 0 & 0 & 0 & 0 & 0.6700 & 0 & 0 \\
\hline & 0 & 0 & 0 & 0 & 0 & 0 & -2.3100 \\
\hline & 0 & 0 & 0 & 0 & 0 & 0 & 0 \\
\hline & 0 & 0 & 0 & 0 & 0 & 0 & 0 \\
\hline & 0 & 0 & 0 & 0 & 0 & 0 & 0 \\
\hline$T_{1}=$ & 0 & 0 & 0 & 0 & 0 & 0 & 0 \\
\hline & 0 & 0 & 0 & 0 & 0 & 0 & 0 \\
\hline & 0 & 0 & 0 & 0 & 0 & 0 & 0 \\
\hline & 0 & 0 & 0 & 0 & 0 & 0 & 0 \\
\hline & 0 & 0 & 0 & 0 & 0 & 0 & -4.6200 \\
\hline & 0 & 0 & 0 & 0 & 0 & 0 & 4.6200 \\
\hline & 0 & -0.3284 & 0 & 0 & 0 & 0 & 0 \\
\hline$A_{2}=$ & 0 & 0.3284 & 0 & 0 & 0 & 0 & 0 \\
\hline & 0 & 0 & 0 & 0 & 0 & 0 & 0 \\
\hline & 0 & 0 & 0 & 0 & -0.6700 & 0 & 0 \\
\hline & 0 & 0 & 0 & 0 & 0.6700 & 0 & 0 \\
\hline & 0 & 0 & 0 & 0 & 0 & 0 & 1.0000 \\
\hline & 0 & 0 & 0 & 0 & 0 & 0 & 1.0000 \\
\hline & 0 & 5.7500 & 0 & 0 & 0 & 0 & 0 \\
\hline$T_{2}=$ & 0 & 5.7500 & 0 & 0 & 0 & 0 & 0 \\
\hline & 0 & 0 & 0 & 0 & 0 & 0 & 0 \\
\hline & 0 & 0 & 0 & 0 & 3.4000 & 0 & 0 \\
\hline & 0 & 0 & 0 & 0 & 3.4000 & 0 & 0 \\
\hline & 0 & 0 & 0 & 0 & 0 & 0 & 0 \\
\hline & 0 & 0 & 0 & 0 & 0 & 0 & 0 \\
\hline & 0 & 0 & 0 & 0 & 0 & 0 & 0 \\
\hline$A_{3}=$ & 0 & -0.3284 & 0 & 0 & 0 & 0 & 0 \\
\hline & 0 & 0.3284 & 0 & 0 & 0 & 0 & 0 \\
\hline & 0 & 0 & 0 & 0 & 0 & 0 & 0 \\
\hline & 0 & 0 & 0 & 0 & 0 & 0 & 0 \\
\hline
\end{tabular}


$T_{3}=\left[\begin{array}{rrrrrrr}0 & 0 & 0 & 0 & 0 & 0 & 0 \\ 0 & 0 & 0 & 0 & 0 & 0 & 0 \\ 0 & 0 & 0 & 0 & 0 & 0 & 0 \\ 0 & 14.750 & 0 & 0 & 0 & 0 & 0 \\ 0 & 14.750 & 0 & 0 & 0 & 0 & 0 \\ 0 & 0 & 0 & 0 & 0 & 0 & 0 \\ 0 & 0 & 0 & 0 & 0 & 0 & 0\end{array}\right]$

From the corresponding equation $F(\lambda)=\operatorname{det}(\boldsymbol{B}(\lambda)-\lambda I)=0$, we obtain the diagram in Figure 4.

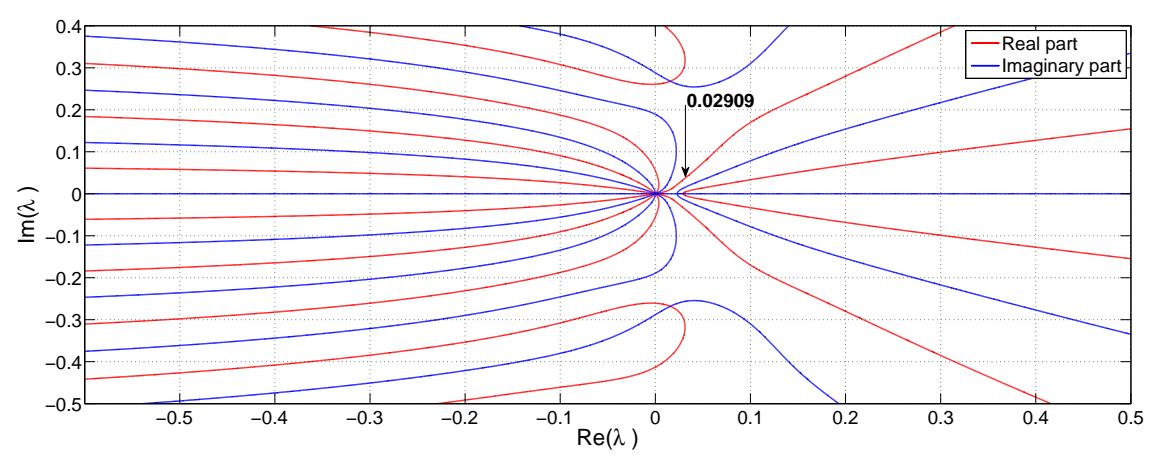

Figure 4: Loci for $F(\lambda)=0$. The real part of $F(\lambda)=0$ is shown in red and the imaginary part of $F(\lambda)=0$ is in blue.

So $\boldsymbol{x} \sim e^{\lambda_{\max } t} \boldsymbol{c}$ : with $\lambda_{\max }=0.02909 h r^{-1}$. Then we just need to find $\boldsymbol{c}=\left(c_{1}, \ldots c_{7}\right)$ such that

$$
\boldsymbol{B}\left(\lambda_{\max }\right) \boldsymbol{c}=\lambda_{\max } \boldsymbol{c} .
$$

In this example

$$
\begin{aligned}
& \left.\boldsymbol{c}=\text { vector }=0.0442: G_{a}\right\} \\
& \left.0.1218: G_{b}\right\} 60.3 \% \text { in the G-phase long-term } \\
& \left.0.4380: G_{c}\right\} \\
& \left.0.2680: S_{a}\right\} \\
& \left.0.0373: S_{b}\right\} 27.5 \% \text { in the S-phase long-term } \\
& \left.0.0809: M_{a}\right\} \\
& \left.0.0097: M_{b}\right\} 12.2 \% \text { in the M-phase long-term }
\end{aligned}
$$

$$
\text { Total }=0.9999 \sim 1
$$




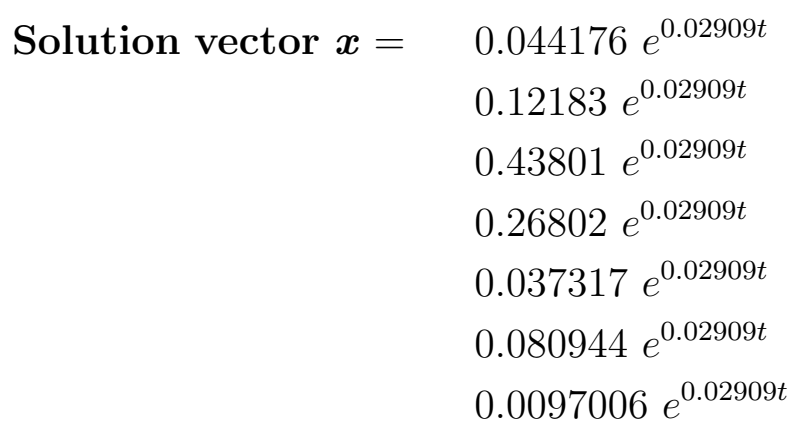

This gives the asymptotic fraction of cells in each of the cells in each compartment.

\section{Conclusion}

This has set out an explicit method of solving systems of the form in equations (5) and extensions of the form in equations (12). The method produces a countable number of solutions which span the set of all solutions. When the dimension of the system is odd (here 7 or 3 ) there is one real positive timeconstant which gives an exponential solution which dominates the long-term behaviour of the solution. In the case study discussed above this enables the determination of the proportion of cells in each of the cell phases for long time. This enables estimates of the effect of the targeted treatments for chemotherapy to be determined.

This has neglected the effects of logistic inputs. The complex roots of $F(\lambda)=0$ give damped oscillatory behaviour.

This seemingly simple example illustrates the fact that delay, and especially multiple-delay systems can explain quite complex behaviour.

Acknowledgements This publication was based on work supported in part by Award No. KUK-C1-013-04, made by King Abdullah University of Science and Technology (KAUST). Thanks are due to PhD candidate at Massey University Mr Ali Ashher Zaidi for the software for the diagrams in Figures 2 and 4, and Research Assistant Miss Andrea Babylon for technical 
assistance. We also thank Dr Kate Simms et al for permission to reproduce figure 3 in this paper.

\section{References}

[1] B. Basse, B. Baguley, E. Marshall, W. Joseph, B. van Brunt, G. Wake, G., and D. Wall, A mathematical model for analysis of the cell cycle in cell lines derived from human tumors, J. Math. Biol. 47 (2003), no. 4, 295-312.

[2] B. Basse, B. Baguley, E. Marshall, W. Joseph, B. van Brunt, G. Wake, G. Wall, and D. Wall, Modelling cell death in human tumour cell lines exposed to the anticancer drug paclitaxel, J. Math. Biol. 49 (2004), no. 4, 329-357.

[3] B. Basse, B. Baguley, E. Marshall, G. Wake, and D. Wall, Modelling the flow of cytometric data obtained from unperturbed human tumour cell lines: parameter fitting and comparison, Bull. Math. Biol. 67 (2005), no. 4, 815-830.

[4] R.E. Bellman and K.L. Cooke, Differential-difference equations, Academic Press, 1963.

[5] J.K. Hale, E.F. Infante, and F.S.P. Tsen, Stability in linear delay equations, J. Math. Anal. Appl. 105 (1985), 533-555.

[6] H.V. Jain and H.M. Byrne, Qualitative analysis of an integro-differential model of periodic chemotherapy, Appl. Math. Lett. 25 (2012), 2132-2136.

[7] L. Johnson, H.M. Byrne, A.E. Willis, and C.A. Laughton, An integrative biological approach to the analysis of tissue culture data: application to the anti-tumour agent RHPS4, Integrative Biology $\mathbf{3}$ (2011), 843-849.

[8] H Rasmussen, G. Wake, and J Donaldson, Analysis of a class of distributed delay logistic differential equations, Mathematical and Computer Modelling 38 (2003), 123-132.

[9] K. Simms, A mathematical model of cell cycle progression applied to breast cancer cell lines, (Doctoral Disertation). School of Mathematical Sciences, University of Adelaide (2011). 
[10] K. Simms, N. Bean, and A. Koerber, A mathematical model of cell cycle progression applied to the MCF-7 breast cancer cell line, Bull. Math. Biol. 74 (2012), 736-767.

\section{Author addresses}

1. G. C. WAKE, Centre for Mathematics in Industry, Institute of Information and Mathematical Sciences, Massey University at Auckland, P.B 102904, North Shore MC, Auckland 0932, NEW ZEALAND.

mailto:g.c.wake@massey.ac.nz

2. H. M BYRNE, Oxford Centre for Collaborative Applied Mathematics, Mathematical Institute, University of Oxford, 24-29 St Giles, Oxford 0X1 3LB, England.

mailto:Helen. Byrne@maths.ox.ac.uk 



\section{RECENT REPORTS}

12/76 A note on heat and mass transfer from a sphere in Stokes flow at low Péclet number

Bell

Byrne

Whiteley

Waters

12/77 Effect of disjoining pressure in a thin film equation with non-

Moulton uniform forcing

Lega

12/78 A Review of Mathematical Models for the Formation of Vascular

Scianna Networks

Bell

Preziosi

12/79 Fast and Accurate Computation of Gauss-Legendre and Gauss-

Hale Jacobi Quadrature Nodes and Weights

Townsend

$12 / 80$ On the spectral distribution of kernel matrices related to radial

Wathen basis functions

Zhu

12/81 Inner product computation for sparse iterative solvers on distributed supercomputer

Zhu

$\mathrm{Gu}$

Liu

12/82 A new pathway for the re-equilibration of micellar surfactant solutions

Griffiths

Breward

Colegate

Dellar

Howell

Bain

12/83 Object-Oriented Paradigms for Modelling Vascular Tumour Growth: a Case Study

Connor

Cooper

Byrne

Maini

McKeever

12/84 Chaste: an open source $\mathrm{C}++$ library for computational physiology and biology

Mirams

Arthurs

Bernabeu

Bordas

Cooper

Corrias

Davit

Dunn

Fletcher

Harvey

Marsh

Osborne

Pathmanathan

Pitt-Francis

Southern

Zemzemi

Gavaghan

12/85 A two-pressure model for slightly compressible single phase flow

Schlackow in bi-structured porous media

Marguerat

Proudfoot

Bähler 
12/89 An experimental and theoretical investigation of particlewall im- Vigolo pacts in a T-junction

Griffiths

Radl

Stone

12/90 Transitions through Critical Temperatures in Nematic Liquid Crystals

Majumdar

Ockendon

Howell

Surovyatkina

12/91 Biaxial defect cores in nematic equilibria: an asymptotic result

Majumdar

Pisante

Henao

12/92 The Three Sphere Swimmer in a Nonlinear Viscoelastic Medium

Curtis

Gaffney

12/93 Diffusion of multiple species with excluded-volume effects

Bruna

Chapman

12/94 The Mechanics of a Chain or Ring of Spherical Magnets

Hall

Vella

Goriely

12/95 On-Lattice Agent-based Simulation of Populations of Cells within

Figueredo the Open-Source Chaste Framework

Joshi

Osborne

Byrne

Owen

12/96 Mathematical Biomedicine and Modeling Avascular Tumor Byrne Growth

12/97 Inference of the genetic network regulating lateral root initiation

Muraro in Arabidopsis thaliana

Voß

Wilson

Bennett

Byrne

De Smet

Hodgman

King

12/98 Axisymmetric bifurcations of thick spherical shells under inflation

deBotton and compression

Bustamante

Dorfmann

Copies of these, and any other OCCAM reports can be obtained from:

Oxford Centre for Collaborative Applied Mathematics

Mathematical Institute 
24 - 29 St Giles'

Oxford

OX1 3LB

England

www.maths.ox.ac.uk/occam 